\title{
Structural characterization of liposomes using integrated methods of HPLC/AF4, UV-Vis absoprtion, Refractive Index, MALLS, DLS, and SAXS
}

\author{
Ting Wei, Hsu, Kuei-Fen Liao ${ }^{1}$, Yi-Qi Yeh, ${ }^{1}$ Orion Shih, ${ }^{1}$ and U-Ser Jeng ${ }^{1,2^{*}}$ \\ ${ }^{1}$ National Synchrotron Radiation Research Center, Hsinchu Science Park, Hsinchu 30076, Taiwan; \\ ${ }^{2}$ Institute of biochemical sciences \& Institute of biological chemistry, Academia Sinica, Nankang, Taipei 115, Taiwan, \\ ${ }^{3}$ Department of Chemical Engineering, National Tsing Hua University, Hsinchu 30013, \\ *usjeng@nsrrc.org.tw
}

Liposome are increasingly better developed as efficient drug carriers. Structural characterization of the functional liposomes with and without drug-uptake and the consequent drug-transport and conditional drug-delivery, is of interest yet not well-stablished. Here, we report an established combined methods using high-performance liquid chromatography(HPLC), asymmetric flow field-flow fractionation (AF4), UV-Vis absorption, refractive index (RI), multi-angle laser light scattering (MALLS), dynamic light scattering (DLS), and small-angle X-ray scattering for structural characterization of liposome solutions. We will demonstrate an example of using the integrated system to successfully determine hydrodynamic radius and its distribution, molecular mass, lipid aggregation number, of a model liposome system. The radius of gyration and detailed bilayer structures of the liposome system are determined using simultaneous small- and wide-angle X-ray scattering, incorporated with HPLC/UVvis/RI, at the high-flux 13A BioSAXS undulator beamline of the $3.0 \mathrm{GeV}$ Taiwan Photon Source. We expect this fast structural characterization system would contribute greatly on drug screening of biomedical industrials.

Keywords: liposome, structure, SAXS 